\title{
Investigation Into Vibration Characteristic in Vibrothermography
}

\author{
Yin $\mathrm{LI}^{1}$, Anbo $\mathrm{MING}^{1 *}$, Ruimin $\mathrm{ZHANG}^{2}$, and Wei ZHANG ${ }^{1}$ \\ ${ }^{1}$ Rocket Force University of Engineering, Xi'an 710025, China \\ ${ }^{2}$ High-tech Institute, Fan Gong-ting South Street on the 12th, Qingzhou 262550, China \\ *Corresponding author: Anbo MINGＩ-mail: Mingab0628@163.com
}

\begin{abstract}
Vibration characteristic plays an important role in vibrothermography, which directly affects the heating during the test. In this work, involving all the contacts in the vibrothermography, the "double-mass-three-spring" model is established to explore the vibration characteristic. The obtained results show that ultrasonic gun vibrates at fundamental frequency (FF), while the specimen vibrates at multi-frequencies including $\mathrm{FF}, 2 \mathrm{FF}, 3 \mathrm{FF}$, and $4 \mathrm{FF}$, which is validated through experimental investigation results. Additionally, the model proposed in this work reveals a high order harmonics in the vibrothermography test and makes the specimen conduct the steady vibration, which indicates that the model is closer to the practical equipment and can ensure the heating efficiency induced by vibration of specimen to improve the detection capability.
\end{abstract}

Keywords: Vibrothermography; vibration characteristic; “double-mass-three-spring” model

Citation: Yin LI, Anbo MING, Ruimin ZHANG, and Wei ZHANG, "Investigation Into Vibration Characteristic in Vibrothermography," Photonic Sensors, 2019, 9(2): 108-114.

\section{Introduction}

Vibrothermography [1, 2], also called as thermosonics, ultrasonic infrared thermography, and acoustic thermography, etc., is viewed as a promising active thermal nondestructive method and attracts extensive attention due to its advantages of easy operation, high efficiency, damage selective imaging, clear visualization, and great adaptability to complex structures, etc., which is suitable for crack detection in the materials. During the vibrothermography test, the ultrasonic generator releases ultrasonic waves into the object, resulting in the ultrasonic vibration of the object. If there is a crack in the object, the dissipative phenomena, such as friction between the faces of crack, will transfer vibrational energy to heat. In this case, we can use an infrared camera to image the heat effect and realize the crack detection. From the process of vibrothermography, it can be known that the principle of vibrothermography mainly contains three aspects: ultrasonic vibration, vibration induced heat, and infrared imaging, among which the ultrasonic vibration is the basis for the latter two and becomes a critical topic of active discussion.

Han et al. [3, 4] first found out the "acoustic chaos" phenomenon, i.e., the presence of multi-frequencies in the vibrational spectrum of specimen that was stimulated by ultrasonic wave with a single frequency and pointed out this phenomenon could enhance the heating effect. Afterwards, both Sovodov [5] and Han [6] started to

Received: 17 July 2018 / Revised: 30 November 2018

(C) The Author(s) 2019. This article is published with open access at Springerlink.com

DOI: $10.1007 / \mathrm{s} 13320-019-0529-\mathrm{x}$

Article type: Regular 
explore the generation mechanism of nonlinear vibration in the vibrothermography test. The former pointed out that the nonlinear vibration was caused by the crack induced material nonlinearity, while the latter thought that the nonlinear vibration existed during the vibrothermography test whether there was a crack or not in the specimen, which was validated a "single-mass" model that supposed the ultrasonic horn as a mass whose length varied harmonically. But, they didn't consider the vibration of specimen. Involving the vibration of specimen, Zheng et al. [7] proposed a "double-mass-double-spring" model for the generation of nonlinear vibration in vibrothermogrphy, in which the ultrasonic horn and the specimen were viewed as a "contact-collision" particle pair. The obtained results illustrate the specimen motion stimulated by the ultrasonic horn and the generation mechanism of nonlinear vibration. On this basis, Chen et al. [8] investigated the nonlinear vibration in the metal plate stimulated by high-power ultrasonic, which pointed out the main reason for generating the nonlinear vibration was the intermittent contact-collision between the ultrasonic horn and plate. Additionally, considering the inevitable energy loss, Feng et al. [9] proposed a mechanical model based on the momentum conservation theorem and investigated the effect of engagement force on vibration characteristics. The obtained results show that the relationship between the engagement force and harmonic order can be described with hyperbola. Tian et al. [10] utilized the finite element method to improve the simulation model to investigate the effect of nonlinear vibration on the detection results. The obtained results show that the generation of nonlinear vibration can remove the standing wave and improve the heating efficiency. Totally, the above literatures available have shown that nonlinear vibrations, including harmonics and acoustic chaos, exist unavoidablely in the vibrothermography test, which can facilitate the understanding of the vibrothermography mechanism to some degree. However, the vibration model in the literatures only contains the contacts between the specimen and clamp, and specimen and ultrasonic gun, without considering the contact between the fastening device and ultrasonic gun.

On this account, it is necessary for a further investigation into vibration characteristic in vibrothermography test. In this work, involving all the contacts, a "double-mass-three-spring" model is proposed for the analysis of the vibration characteristic in vibrothermogrphy, which is validated by experiments.

\section{Methods section}

To investigate the vibration characteristic comprehensively, the "double-mass-three-spring" vibration model is first proposed according to the contact state during the vibrothermography test. Then, the steel plate with fatigue crack is used to conduct the experiment for validating the proposed model.

\section{1 "Double-mass-three-spring" model}

Actually, the ultrasonic gun is generally fixed by a fastening device to facilitate to stimulate the specimen during the vibrothermgoraphy test, in this case, the two ends of ultrasonic gun are both restrictive, not a side free, the other restrictive, as shown in Fig. 1. Thus, the contact between the ultrasonic gun and fastening device should be considered in the analysis of the vibration characteristic.

As shown in Fig. 1, the ultrasonic gun and the specimen are fastened by the fastening device and clamp, respectively. Additionally, the contact force $F_{0}$ between the ultrasonic gun and the specimen is determined by the fastening device. During the vibrothermography test, the generator releases sinusoidal ultrasonic wave, in the form of $A \sin (2 \pi f)$, into the specimen through the ultrasonic gun to induce the vibration of the specimen. Thus, we can conclude that there are three contacts during the vibrothermography test, i.e., the contact between the 
fastening device and ultrasonic gun, ultrasonic gun and specimen, and specimen and clamp. Involving all the contacts, a physical model, as shown in Fig. 2, is established to investigate the vibration characteristic, where the ultrasonic horn is equivalent to a particle $M_{1}$ with a displacement of $X_{1}$, the specimen is equivalent to a particle $M_{2}$ with a displacement of $X_{2}$, and the above-mentioned contacts are equivalent to the springs with the stiffness coefficients of $K_{1}, K_{2}$, and $K_{3}$ and damping coefficients of $R_{1}, R_{2}$, and $R_{3}$, respectively. It is noted that the spring $\left(K_{1}, R_{1}\right)$ is inextensible because the ultrasonic horn and specimen can be separated in the actual vibrothermography test.

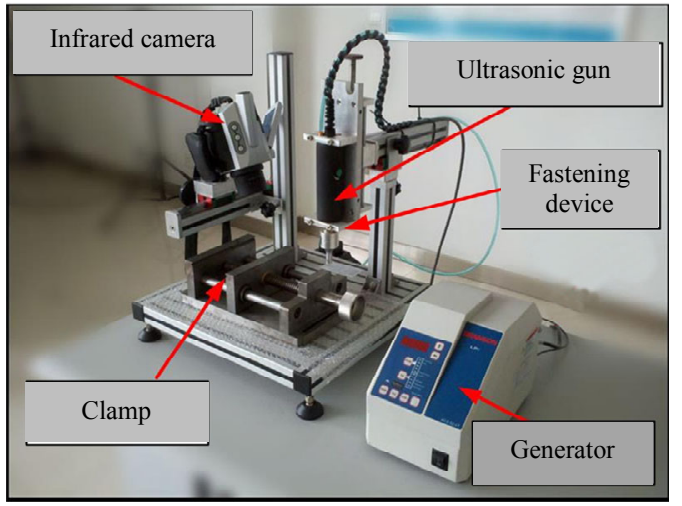

(a)

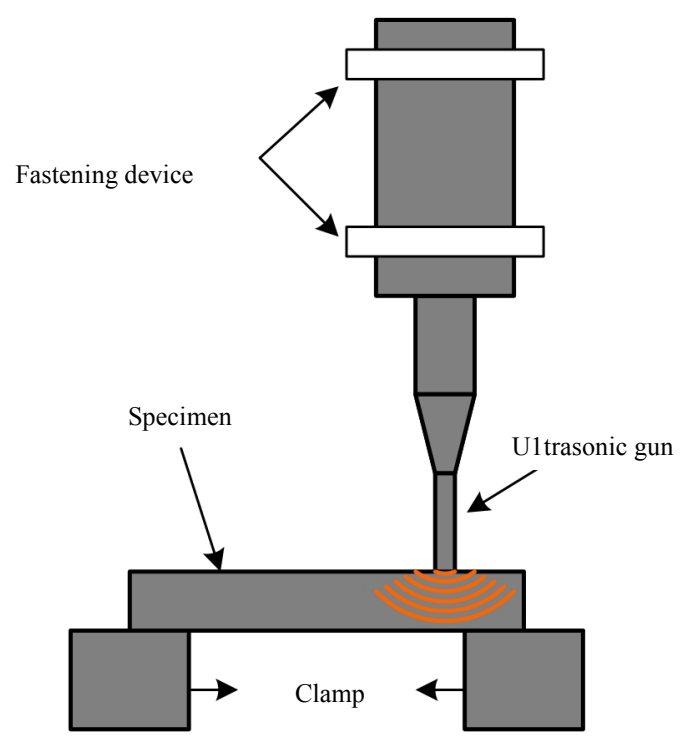

(b)

Fig. 1 Vibrothermography test devices: (a) the actual device photo and (b) the simplified sketch map.
From the physical model in Fig. 2, we can deduce the motion equations of $M_{1}$ and $M_{2}$ based on the Newton second law.

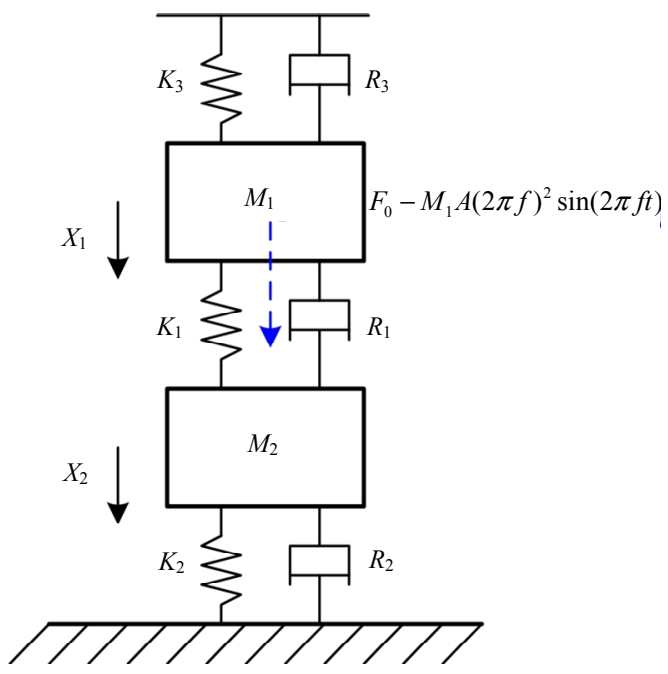

Fig. 2 Physical model of nonlinear vibration.

In the case of $X_{1} \geq X_{2}$, i.e. $M_{1}$ is contacted with $M_{2}$, the motion equations can be deduced as follows:

$$
\left\{\begin{array}{rl}
M_{1} \ddot{X}_{1}= & F_{0}-M_{1} A(2 \pi f)^{2} \sin (2 \pi f t)-K_{1}\left(X_{1}-X_{2}\right) \\
& -R_{1}\left(\dot{X}_{1}-\dot{X}_{2}\right)-K_{3} X_{1}-R_{3} \dot{X}_{1} \\
M_{2} \ddot{X}_{2}= & K_{1}\left(X_{1}-X_{2}\right)+R_{1}\left(\dot{X}_{1}-\dot{X}_{2}\right)-K_{2} X_{2}-R_{2} \dot{X}_{2}
\end{array} .\right.
$$

In the case of $X_{1}<X_{2}$, i.e. $M_{1}$ is separated from $M_{2}$, the motion equations can be deduced as follows:

$$
\left\{\begin{array}{l}
M_{1} \ddot{X}_{1}=F_{0}-M_{1} A(2 \pi f)^{2} \sin (2 \pi f t)-K_{3} X_{1}-R_{3} \dot{X}_{1} \\
M_{2} \ddot{X}_{2}=-K_{2} X_{2}-R_{2} \dot{X}_{2}
\end{array} .\right.
$$

With the initial condition of $X_{1}=X_{2}=\dot{X}_{1}=\dot{X}_{2}=0$, the corresponding parameters for solving the above equations are listed as: $M_{1}=1 \mathrm{~kg}, M_{2}=0.5 \mathrm{~kg}, F_{0}=200 \mathrm{~N}, A=70 \mu \mathrm{m}, f=$ $40 \mathrm{kHz}, K_{1}=10^{9} \mathrm{~N} / \mathrm{m}, K_{2}=3 \times 10^{6} \mathrm{~N} / \mathrm{m}, K_{3}=10^{10} \mathrm{~N} / \mathrm{m}$, $R_{1}=10^{5} \mathrm{~N} \mathrm{~s} / \mathrm{m}, R_{2}=100 \mathrm{~N} \mathrm{~s} / \mathrm{m}$, and $R_{3}=10 \mathrm{~N} \mathrm{~s} / \mathrm{m}$. It is noted that the parameters used in this work are partly obtained by [7,9] and partly obtained by experiments.

\subsection{Experimental validation}

For validating the vibration characteristic of the specimen obtained by the above model, the experimental investigation is carried out in this 
section. The specimen studied in the experiment is steel plate with the size of $140 \mathrm{~mm} \times 30 \mathrm{~mm} \times 3 \mathrm{~mm}$, and the ultrasonic stimulation device is produced by Branson company with the type of 2000LPt, which can release the ultrasonic wave with the frequency of $40 \mathrm{kHz}$ to stimulate the steel plate. A laser vibrometer, produced by KEYENCE Company, with the type of LK-G5000, with the accuracy of $\pm 0.02 \%$ and the frequency of $392 \mathrm{kHz}$, is used to measure the displacement of the specimen in the vibrothermography test. The simplified sketch map of the experimental device is shown in Fig. 3.

During the experiment, the continuous ultrasonic wave is released to stimulate the steel plate. When the steel plate reaches to the stable vibration state, the laser vibrometer starts to gather the displacement of measurement point, which is located close to the excitation point between the ultrasonic gun and specimen.

Fig. 3 Simplified sketch map of the experimental device.

\section{Results and discussion}

Figure 4 shows the vibration displacement of the ultrasonic gun and specimen obtained by the "double-mass-three-spring" model.

As shown in Fig. 4, the ultrasonic gun vibrates in a sinusoidal manner due to the sinusoidal excitation of the generator, which leads to the periodic vibration of specimen. When the ultrasonic gun contacts with the specimen, the displacement of the specimen gradually increases under the action of the spring $\left(K_{1}, R_{1}\right)$, while the ultrasonic gun separates from the specimen, the displacement of the specimen gradually decreases under the action of the spring $\left(K_{2}, R_{2}\right)$. To explore the vibration characteristic of the ultrasonic gun and specimen, the frequency-spectrum analysis is carried out. Figure 5 shows the frequency-spectrum curve of the ultrasonic gun and specimen, where we can know that the ultrasonic gun vibrates at the fundamental frequency (FF) of $40 \mathrm{kHz}$, while the specimen vibrates at multi-frequencies including $\mathrm{FF}(40 \mathrm{kHz})$, $2 \mathrm{FF}(80 \mathrm{kHz}), 3 \mathrm{FF}(120 \mathrm{kHz})$, and $4 \mathrm{FF}(160 \mathrm{kHz})$.

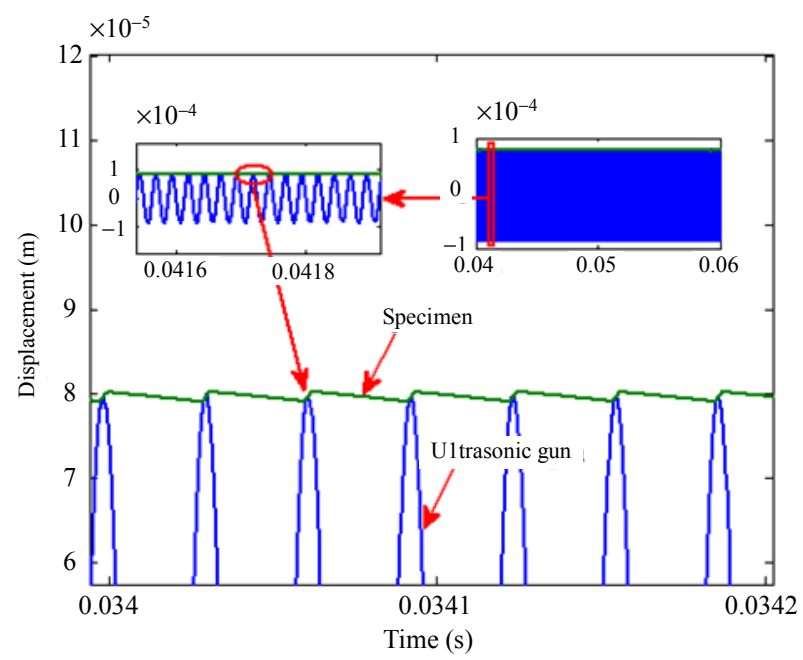

Fig. 4 Vibration displacement of the ultrasonic gun and specimen obtained by the model.

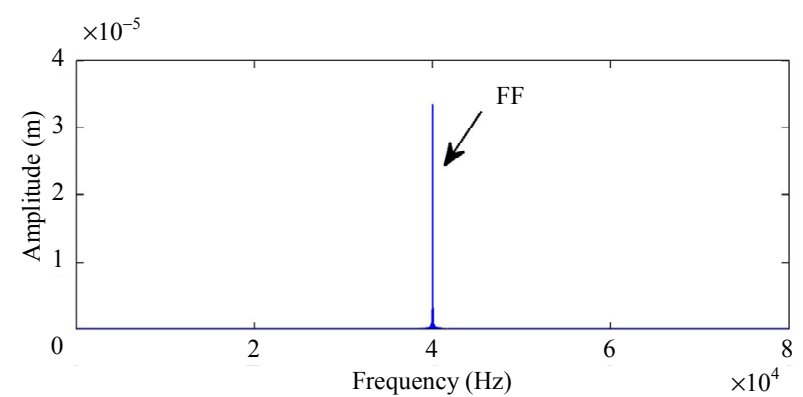

(a)

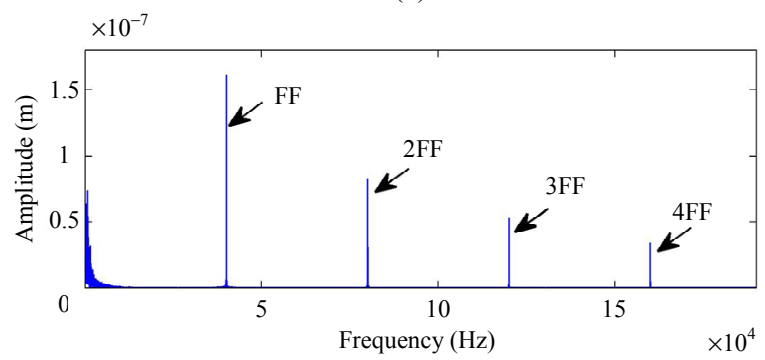

(b)

Fig. 5 Frequency-spectrum curve obtained by the model: (a) ultrasonic gun and (b) specimen. 
To validate the results obtained by the model, the vibration displacements of 4 measurement points which are randomly selected with the serial number
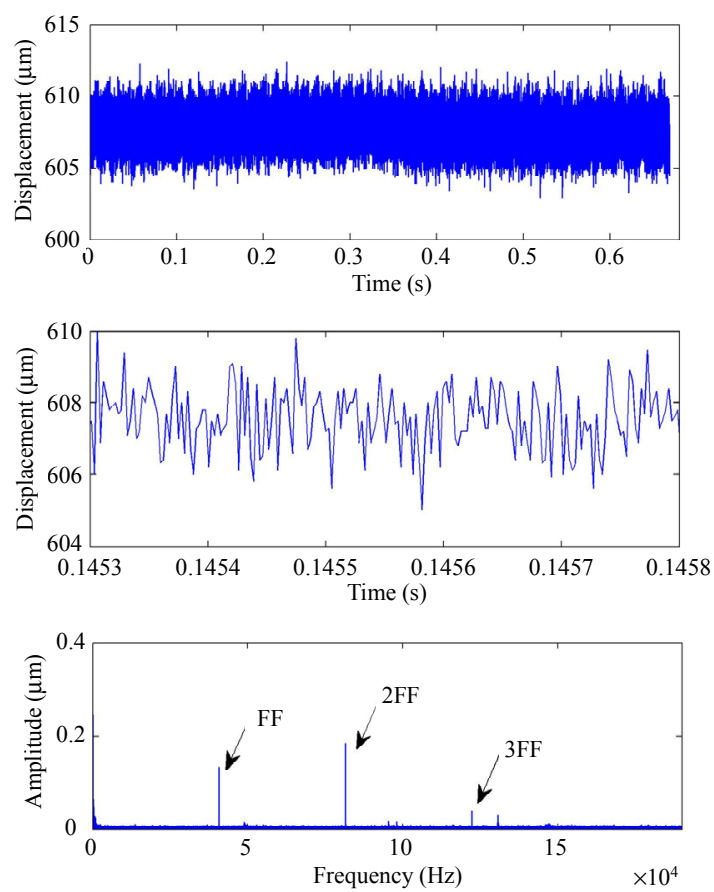

(a)
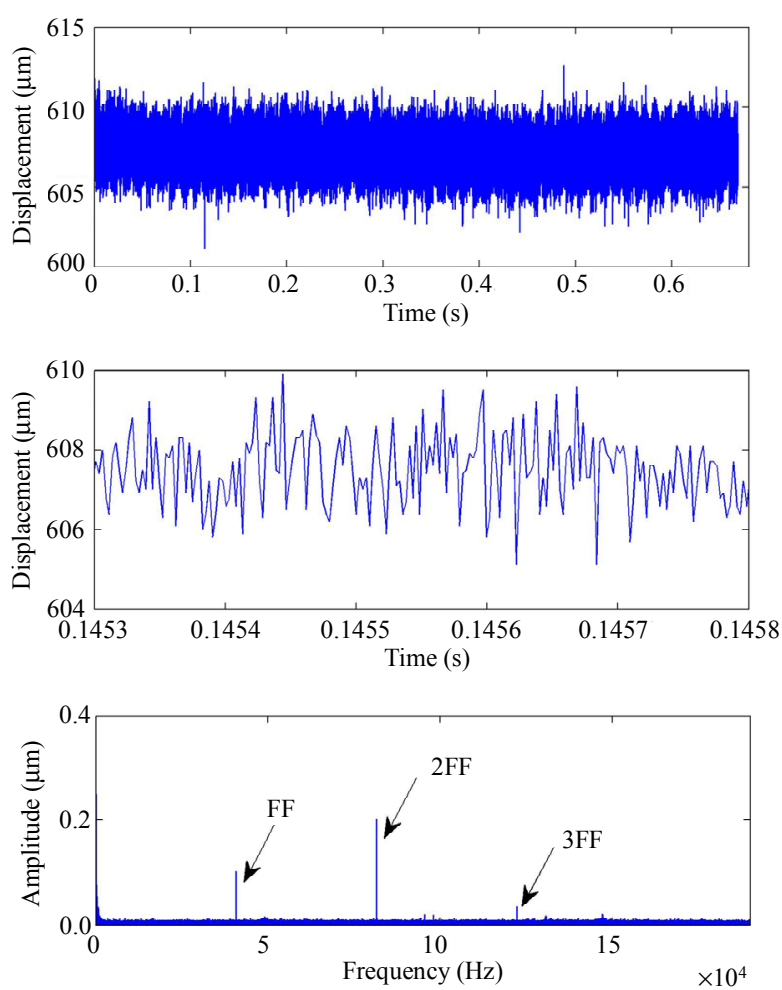

(c) of $1 \#-4 \#$ are gathered by using a laser vibrometer. Figure 6 shows the vibration characteristic of 4 measurement points.
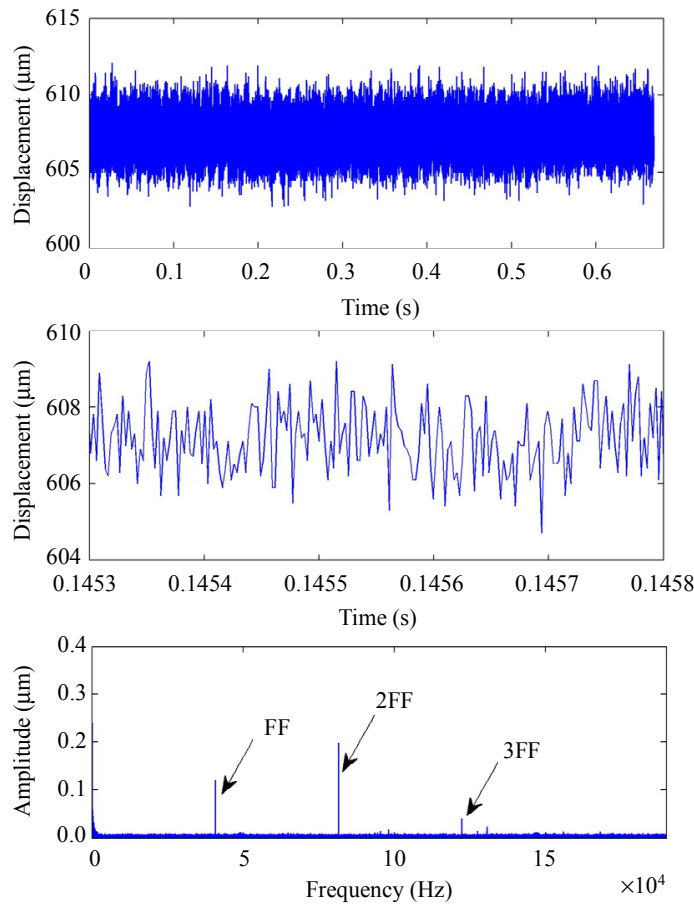

(b)
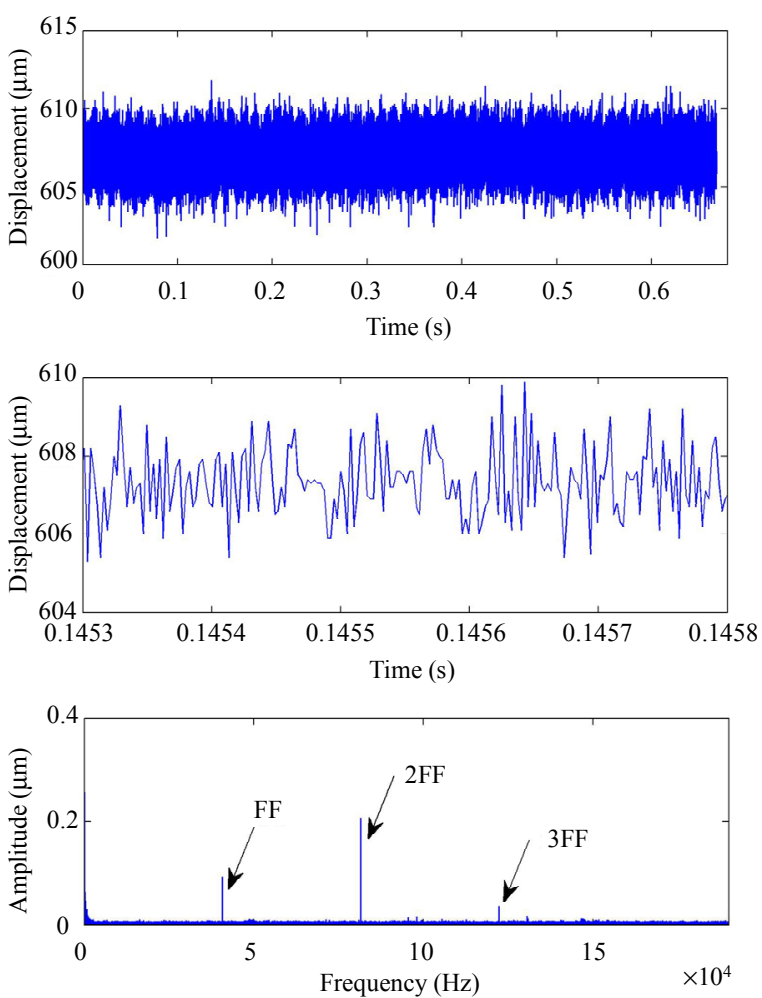

(d)

Fig. 6 Vibration displacement during the gather time of $0 \mathrm{~s}-0.68 \mathrm{~s}$ and $0.1453 \mathrm{~s}-0.1458 \mathrm{~s}$ and the frequency-spectrum curve of the measurement points: (a) $1 \#$, (b) $2 \#$, (c) $3 \#$, and (d) $4 \#$. 
To clearly observe the vibration displacement, the local vibration displacement between $0.1453 \mathrm{~s}$ and $0.1458 \mathrm{~s}$ is extracted, from which we can find 4 measurement points vibrate inconsistently. But the measurement points vibrate at the same frequency including FF (40 kHz), 2FF ( $80 \mathrm{kHz})$, and 3FF (120 $\mathrm{kHz}$ ), which are approximately agreement with that obtained by the model and proves the validation of the model.

To explore the advantages of the model, the comparative analysis between the results obtained by the model in this work and by that in [7] is carried out. Two models are calculated based on the parameters in this work, and the corresponding results are shown in Fig. 7.

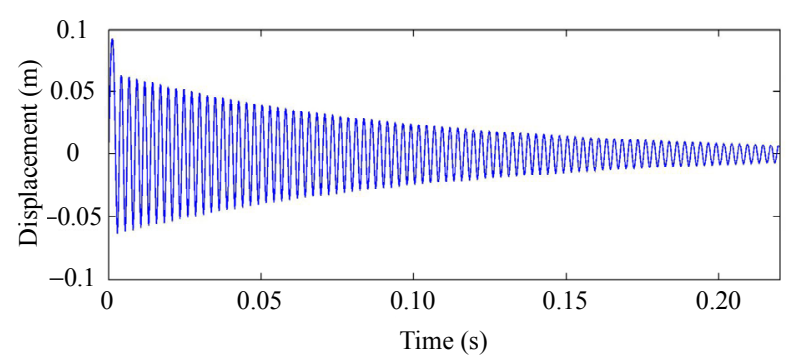

(a)

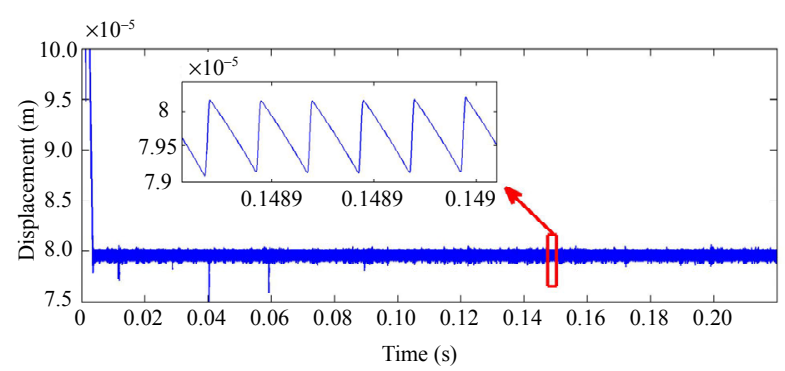

(b)

Fig. 7 Vibration displacement of the specimen: (a) obtained by the model in [7] and (b) obtained by the model in this work.

As known in Fig. 7, the response obtained with the model in [7] is gradually damped and indicates that only transient responses are obtained by the model. Comparatively, the vibration obtained by our model is a steady response excited by the ultrasonic gun, which demonstrates the model in this work can ensure the heating efficiency induced by the vibration of the specimen and improve the detection capability. In fact, the ultrasonic gun is continuously used to excite the specimen, and the collected vibration is the steady response of the specimen. Additionally, our model is to reveal the high order harmonics in the vibrothermography test, which is not observed in [7]. And such phenomenon indicates that our model is closer to the practical equipment than that in [7]. In this case, our model is apparently more reasonable than that in [7].

\section{Conclusions}

Involving all the contacts in the vibrothermography test, a "double-mass-three-spring" model for analyzing the vibration characteristic is established and solved. The obtained results show that the ultrasonic gun vibrates only at the fundamental frequency while the specimen vibrates at multi-frequencies, which is validated by the experimental results. Compared with the model in [7], the model proposed in this work reveals the high order harmonics in the vibrothermography test and makes the specimen conduct the steady vibration, which indicates the model is closer to the practical equipment and can ensure the heating efficiency induced by the vibration of the specimen to improve the detection capability. Anyone can apply this model within the NDT (Nondestructive Testing)-world and profit from it.

\section{Acknowledgement}

This work was supported by the National Natural Science Foundation of China (Grant Nos. 51605481, 51575516, and 51505486).

The authors would like to thank associate Prof. Zheng-wei YANG with Rocket Force University of Engineering, for THE help in revised manuscript.

Open Access This article is distributed under the terms of the Creative Commons Attribution 4.0 International License (http://creativecommons.org/licenses/by/4.0/), which permits unrestricted use, distribution, and reproduction in any medium, provided you give appropriate credit to the original author(s) and the source, provide a link to the Creative Commons license, and indicate if changes were made. 


\section{References}

[1] A. S. Rizi, S. Hedayatrasa, X. Maldgue, and T. Vukhanh, "FEM modeling of ultrasonic vibrothermography of a damaged plate and qualitative study of heating mechanisms," Infrared Physics \& Technology, 2013, 61(5): 101-110.

[2] R. Montanini and F. Freni, "Correlation between vibrational mode shapes and viscoelastic heat generation in vibrothermography," NDT \& E International, 2013, 58: 43-48.

[3] X. Y. Han, W. Li, Z. Zeng, L. D. Favro, and R. L. Thomas, "Acoustic chaos and sonic infrared imaging," Applied Physics Letters, 2002, 81(17): 3188-3190.

[4] X. Y. Han, Z. Zeng, W. Li, M. S. Islam, J. P. Lu, V. Loggins, et al., "Acoustic chaos for enhanced detectability of cracks by sonic infrared imaging," Journal of Applied Physics, 2004, 95(7): 3792-3797.

[5] I. Solodov, J. Wackerl, K. Pfleiderer, and G. Busser, "Nonlinear self-modulation and subharmonic acoustic spectroscopy for damage detection and location,"
Applied Physics Letters, 2004, 84(26): 5386-5388.

[6] X. Y. Han, V. Loggoms, Z. Zeng, L. D. Favro, and R. L. Thomas, "Mechanical model for the generation of acoustic chaos in sonic infrared imaging," Applied Physics Letters, 2004, 85(8): 1332-1334.

[7] K. Zheng, H. Zhang, S. Y. Zhang, and L. Fan, "A dynamical model of subharmonic generation in ultrasonic infrared thermography," Ultrasonics, 2006, 44(8): e1343-e1347.

[8] Z. J. Chen, S. Y. Zhang, and K. Zheng, "Nonlinear vibration in metal plate excited by high-power ultrasonic pulses," Acta Physica Sinica, 2010, 59(6): 4071-4083.

[9] F. Z. Feng, C. S. Zhang, Q. X. Min, and P. F. Wang, "Effect of engagement force on vibration characteristics in sonic IR imaging," Ultrasonics, 2015, 56: 473-476.

[10] G. Tian, Z. W. Yang, J. T. Zhu, W. Zhang, and W. Y. Luo, "Vibration characteristic and acoustic chaos analysis of ultrasonic infrared thermal wave test," Infrared and Laser Engineering, 2016, 45(3): 0304003-1-0304003-6. 